CONFORMAL GEOMETRY AND DYNAMICS

An Electronic Journal of the American Mathematical Society

Volume 10, Pages 125-135 (July 6, 2006)

S $1088-4173(06) 00139-1$

\title{
SOME RATIONAL MAPS WHOSE JULIA SETS ARE NOT LOCALLY CONNECTED
}

\author{
P. ROESCH
}

\begin{abstract}
We describe examples of rational maps which are not topologically conjugate to a polynomial and whose Julia sets are connected but not locally connected.
\end{abstract}

\section{INTRODUCTION AND MOTIVATIONS}

The dynamics of a rational map $f$ acting on $\widehat{\mathbf{C}}$ is concentrated on its Julia set which is (by definition) the minimal compact set invariant by $f$ and $f^{-1}$ containing at least three points. The question of local connectivity of the Julia set is important when one wants to give a model of the dynamics on some part of this limit set (using Carathéodory's Theorem for instance), since on its complement, the Fatou set, the dynamics is well understood. This question of local connectivity also concerns the limit set of Kleinian groups, which is the minimal compact set invariant by the group and which contains at least three points (in order to consider only non-elementary Kleinian groups). There is a well-known analogy between theory and results for these two dynamical systems on $\widehat{\mathbf{C}}=\partial \mathbf{B}^{3}$ which is presented in the dictionary of Sullivan (see [Su, McM1]). All known examples of Kleinian groups have locally connected limit sets (provided they are connected) and there is a model for the action of the group on its limit set; see AnMa, McM2, Min. On the other hand, there are several examples of non-locally connected Julia sets for polynomials; for instance Douady, and Sullivan proved (see $\mathbf{S u}$ ) that any polynomial which has a Cremer periodic point has a non-locally connected Julia set; there are also several examples among infinitely renormalizable polynomials (see [Mi2, [So]). This is not the case for rational maps. Indeed, in Ro we give a family of rational maps for which all Julia sets are locally connected. This family contains rational maps with a periodic Cremer point but also rational maps which are infinitely renormalizable near a critical point. In both cases, the Julia set of the rational map contains an homeomorphic image of a non-locally connected Julia set of a quadratic polynomial. It is itself locally connected. What happens is that the Julia set is reconnected by the boundary of small Fatou components that insert themselves "between the hairs". So, two questions arise naturally. First, are the polynomials very particular cases that do not fit in Sullivan's dictionary? More precisely, if we define genuine rational maps as rational maps that are not topologically conjugated on any neighborhood of the Julia set to a polynomial, the question is : Does there exist genuine

Received by the editors May 11, 2005 and, in revised form, April 7, 2006.

2000 Mathematics Subject Classification. Primary 37F50; Secondary 37F10.

Research partially supported by the Morningside Center of Mathematics in Beijing.

(C)2006 American Mathematical Society

Reverts to public domain 28 years from publication 
rational maps for which the Julia set is not locally connected? Secondly, is the Julia set of a genuine rational map having a quadratic-like restriction always reconnected as in $[\mathrm{Ro}]$ ?

In this article we give a very simple example that answers both questions.

Theorem 1. There exist parameters $a \in \mathbf{C} \backslash\{ \pm 1,0\}$ such that the map $f_{a}(z)=$ $z^{3} \frac{z-a}{1-a z}$ is a genuine rational map having a connected but not locally connected Julia set. Moreover, $f_{a}$ is renormalizable near each critical point and the Julia set of the renormalized polynomial is not locally connected.

We also describe very roughly the example of Ghys, Herman, Douady, and Sullivan which gives certainly the first genuine rational map with non-locally connected Julia set. However this example is not renormalizable so does not answer the second question.

Recall that a rational map is renormalizable if some iterate of it admits a restriction which is quadratic like with connected Julia set.

Definition 0.1. A holomorphic map $f: U^{\prime} \rightarrow U$ with $U=f\left(U^{\prime}\right) \supset \overline{U^{\prime}}$ which is proper and of degree 2 is called a quadratic-like map (after DoHu2]) if $U$ and $U^{\prime}$ are topological disks. Its filled Julia set is the set $K_{f}=\bigcap f^{-n}(U)$ and its Julia set is the boundary $\partial K_{f}$.

Recall that a periodic point $x$ of period $k$ with multiplier $\lambda=\left(f^{k}\right)^{\prime}(x)$ is called respectively repelling, attracting, or indifferent if $|\lambda|>1,|\lambda|<1$, or $|\lambda|=1$. There are different kinds of indifferent cycles depending on $\theta \in \mathbf{R}$ where $\lambda=e^{2 i \pi \theta}$. If $\theta \in \mathbf{Q}$, then the point $x$ belongs to $J(f)$ and is called a parabolic point. If $\theta \in \mathbf{R} \backslash \mathbf{Q}$ with $x \in J(f)$, then the map is not linearizable near $x$ and $x$ is called a Cremer point. If $\theta \in \mathbf{R} \backslash \mathbf{Q}$ with $x \notin J(f)$, then the map is conjugated to the rotation $z \mapsto e^{2 i \pi \theta} z$ on a disk containing $x$; the maximal domain of linearization is a disk called a Siegel disk and $x$ is called a Siegel point.

\section{The example of Ghys, Herman, Douady and Sullivan}

Theorem 1.1 (Ghys, Herman, Douady, Sullivan). There exist quadratic polynomials of the form $P(z)=e^{2 i \pi \theta} z+z^{2}$ such that 0 is a Siegel fixed point and the Julia set $J(P)$ is connected but not locally connected.

The proof of Theorem 1.1 can be found in $\mathrm{Do}, \mathrm{Gh}, \mathrm{He}, \mathrm{Su}, \mathrm{Za}$. We will sketch it but we will give the details of the construction of E. Ghys in order to deduce the following corollary.

Proposition 1.2. There exist genuine rational maps whose Julia set is connected but not locally connected. Furthermore, they are not renormalizable.

For $a>3$, the Blaschke fraction $f_{a}(z)=z^{2} \frac{z-a}{1-\bar{a} z}$ induces a $\mathbf{R}$-analytic diffeomorphism of $\mathbf{S}^{1}$. Indeed, except 0 and $\infty$, the critical points are real, distinct and symmetric with respect to $\mathbf{S}^{1}$ so that there is an inverse image of $\mathbf{S}^{1}$ in $\mathbf{D}$ which surrounds the pole $1 / \bar{a}$ and another inverse image outside $\mathbf{D}$ surrounding the zero $a$. Multiplying by some $e^{2 i \pi t}$ we get a new map $f_{a, t}=e^{2 i \pi t} f_{a}$, which restricts to a diffeomorphism of $\mathbf{S}^{1}$ with rotation number denoted by $\alpha(t)$. If $\alpha(t) \in \mathbf{R} \backslash \mathbf{Q}$, the map $f_{a, t}$ is conjugated by an homeomorphism $h_{a, t}$ of $\mathbf{S}^{1}$ to the rotation $R_{\alpha(t)}$ (by Denjoy's Theorem). This conjugacy is unique up to composition with a rotation. 
Lemma 1.3 (Ghys). If the conjugacy $h_{a, t}$ is quasi-symmetric but not $\mathbf{R}$-analytic, the polynomial $P(z)=e^{2 i \pi \alpha(t)} z+z^{2}$ has a Siegel disk whose boundary is a quasicircle which does not contain the critical point.

The following work of M. Herman $\mathrm{He}$ provides examples of such conjugacies.

Lemma 1.4 (Herman). For $a>3$, there exists $t \in \mathbf{R}$ such that $\alpha(t) \in \mathbf{R} \backslash \mathbf{Q}$ and $h_{a, t}$ is quasi-symmetric but not $\mathcal{C}^{2}$.

Theorem 1.1 follows from Lemma 1.3, Lemma 1.4 and Lemma 1.5 in which we take for $C$ the boundary of the Siegel disk of $P$ and $p=\infty$. We give the proof of Lemma 1.5 since we will use it several times in the article.

Lemma 1.5 (Douady, Sullivan). Let $f$ be a rational map with a super-attracting fixed point $p$. Let $B(p)$ denote the Fatou component containing $p$. If there is a non-empty compact set $C \subset \partial B(p)$ such that $\left.f\right|_{C}$ is a bijection of $C$, then either $J(f)$ is not locally connected or $C$ is a finite union of parabolic or repelling periodic cycles.

Proof. We reproduce the proof of non-local connectivity given by Douady and Sullivan in the case of polynomials with Cremer points (see [Mi1, Su]). We assume for contradiction that the Julia set is locally connected so that in particular $B(p)$ is simply connected and the boundary of $B(p)$ is also locally connected. The map $f$ is conjugated on $B(p)$ to $z \mapsto z^{n}$ for some $n>1$ so by Caratheodory's theorem we can extend continuously the linearization $\phi$ to $\bar{\phi}: \overline{\mathbf{D}} \rightarrow \bar{B}(p)$. The set $\Theta \subset \mathbf{R} / \mathbf{Z}$ of angles $\theta$ such that $\bar{\phi}\left(e^{2 i \pi \theta}\right) \in C$ is stable by the multiplication $\tau_{n}(\theta)=n \theta$. Moreover, since $\left.f\right|_{C}$ is a bijection of $C$, the map $\tau_{n}$ is a bijection of $\Theta$. Since $\Theta$ is a compact subset of $\mathbf{R} / \mathbf{Z}$ and the multiplication by $n$ is expanding, $\Theta$ is reduced to a finite number of points so that the angles in $\Theta$ are all periodic under multiplication by $n$. Therefore $C$ consists in periodic cycles which are either parabolic or repelling by the Snail Lemma (see Mi1]).

Proof of Proposition 1.2. Consider a map $f_{a, t}$ with $t$ given by Herman's Lemma. Let's prove that it satisfies Proposition 1.2, It is a genuine rational map and the proof of this fact is exactly the same as in Lemma 2.6, so we refer to it. To see that the Julia set $J\left(f_{a, t}\right)$ is not locally connected we describe how one goes from $f_{a, t}$ to $P$. This is done by Ghys' Lemma via a surgery process on $f_{a, t}$ as follows. The conjugacy $h_{a, t}$ extends to a $K$-quasi-conformal homeomorphism $H$ of $\mathbf{D}$ by Ahlfors-Beurling's Theorem. The map $T: \widehat{\mathbf{C}} \rightarrow \widehat{\mathbf{C}}$ defined by $T(z)=$ $f_{a, t}(z)$ for $|z| \geq 1$ and $T(z)=H^{-1} \circ R_{\alpha(t)} \circ H(z)$ on $\overline{\mathbf{D}}$, is continuous but not holomorphic. Following E. Ghys, we are going to change the complex structure in order to construct the desired polynomial. Let $\mu(z)$ be the Beltrami form on $\mathbf{D}$ defined by $\mu=\left(\frac{\partial H}{\bar{\partial} z} / \frac{\partial H}{\partial z}\right) \frac{d \bar{z}}{d z}$. Then $\mu$ is invariant by $T$ and has $L^{\infty}$ norm less or equal to $k=\frac{K-1}{K+1}$. For $n \geq 1$, one extends $\mu$ on $T^{-n}(\mathbf{D})$ by the pull back $\left(T^{n}\right)^{*} \mu$ of the form defined on $\mathbf{D}$, and one sets $\mu=0$ on $\mathbf{C} \backslash \bigcup_{n \geq 0} T^{-n}(\mathbf{D})$. This Beltrami form is clearly invariant by $T$ and still satisfies $\|\mu\|_{\infty} \leq k<1$. By Ahlfors-Bers, Morrey, Boyarski's Theorem, there exists a $K$-quasi-conformal homeomorphism $G: \widehat{\mathbf{C}} \rightarrow \widehat{\mathbf{C}}$ integrating $\mu$ and tangent to identity near $\infty$. Therefore $P=G \circ T \circ G^{-1}$ is a 
rational map, since it preserves the standard complex structure of $\widehat{\mathbf{C}}$. This rational map $P$ has the following properties: it has no poles, and the point $\infty$ is fixed and critical. Therefore $P$ is a quadratic polynomial. Moreover, $P$ is conjugated on $G(\mathbf{D})$ by $H \circ G^{-1}$ to the rotation $R_{\alpha(t)}$ in $\mathbf{D}$, so that $G(0)$ is a Siegel fixed point. Moreover $G(\mathbf{D})$ is exactly the Siegel disk. Indeed, if there is a topological disk $\Delta$ invariant by $T$ larger than $\mathbf{D}$, then the annulus $\Delta \backslash \mathbf{D}$ is invariant by $P$. By the Schwarz reflection principle, it follows that $P$ is analytically conjugated to a rotation on the annulus $\Delta \cap \iota(\Delta)$ where $\iota(z)=1 / z$ (the rotations are the only automorphisms of annuli). Since this annulus contains $\mathbf{S}^{1}$, this contradicts the fact that $h_{a, t}$, which is not $\mathbf{R}$-analytic, is unique up to composition with a rotation. Finally, as $G$ is an homeomorphism it preserves the local degree so that $P$ has no critical point on the boundary of the Siegel disk $\partial G(\mathbf{D})$. This achieves the proof of Ghys' Lemma.

Proposition 1.2 follows now since the basins of $\infty$ - i.e., the connected component containing $\infty$ of the complement of the Julia set - correspond to each other. More precisely, let $B_{a, t}(\infty)$, resp. $B(\infty)$ denote the basin of $\infty$ for $f_{a, t}$, resp. for $P$; then clearly $G\left(B_{a, t}(\infty)\right)=B(\infty)$ and, since $G$ is an homeomorphism, $G\left(\partial B_{a, t}(\infty)\right)=\partial B(\infty)$. It is a classical fact that if the Julia set of $f_{a, t}$ is locally connected, then $\partial B_{a, t}(\infty)$ (see $[\mathrm{Wh}$ ) is also locally connected. Its image $\partial B(\infty)$, which is $J(P)$, is then also locally connected. This contradicts Theorem 1.1.

Now we are going to prove by contradiction that $f_{a, t}$ is not renormalizable near the critical point $\omega_{+}$in $\mathbf{C} \backslash \mathbf{D}$ (the argument is analogous for the symmetric critical point $\left.\omega_{-}=1 / \omega_{+}\right)$. Assuming the contrary, there exist $k \geq 1$ and disks $U^{\prime}, U$ with $\overline{U^{\prime}} \subset U$ such that $f_{a, t}^{k}: U^{\prime} \rightarrow U$ is a degree two covering and $f_{a, t}^{k n}\left(\omega_{+}\right) \in U^{\prime}$ for every $n \geq 0$. This property also holds when one replaces $U^{\prime}$ by any preimage $U_{i}^{\prime}$ of $U^{\prime}$ by $f_{a, t}^{k i}$ which contains $\omega_{+}$and $U$ by $U_{i}=f_{a, t}^{k}\left(U_{i}^{\prime}\right)$. Note that $\mathbf{S}^{1}$ is contained in every $U_{i}^{\prime}$. Indeed, for the polynomial $P$, the boundary of the Siegel disk is contained in the accumulation set of the forward orbit of 0 (which is the critical point of $P$ ). Therefore $\mathbf{S}^{1}$, its image by $G^{-1}$, is in the accumulation set of the forward orbit of $\omega_{+}=G^{-1}(0)$. So for any point $x \in \mathbf{S}^{1}$, one can extract from any sequence $\left(f_{a, t}^{n_{j}}\left(\omega_{+}\right)\right)_{j \in \mathbf{N}}$ converging to $x$ a subsequence of the form $\left(f_{a, t}^{m_{j} k+r}\left(\omega_{+}\right)\right)_{j \in \mathbf{N}}$ with $0 \leq r<k$. Therefore $f_{a, t}^{r}\left(U_{n}^{\prime}\right)$ intersects $\mathbf{S}^{1}$ for any $n \geq 0$. Moreover, $\mathbf{S}^{1}$ is contained in $U_{i}^{\prime}$ since the orbit of any point is dense in $\mathbf{S}^{1}$. As $U_{i}^{\prime}$ is simply connected it contains either $\overline{\mathbf{D}}$ or $\widehat{\mathbf{C}} \backslash \mathbf{D}$ so that its image $f_{a, t}\left(U_{i}^{\prime}\right)=\widehat{\mathbf{C}}$ and thus $U_{i-1}^{\prime}=\widehat{\mathbf{C}}$ also. This contradicts the fact that $f^{k}: U^{\prime} \rightarrow U$ is a degree 2 proper map.

If the conjugacy $h_{a, t}$ is not quasi-symmetric anymore, it is not possible to make the surgery, as it has been done. Nevertheless, using Lemma 1.7 and Lemma 1.5. we obtain the following result.

Proposition 1.6. For $a>3$ and any $t \in \mathbf{R}$ such that the rotation number $\alpha(t)$ of $f_{a, t}$ is irrational, the Julia set $J\left(f_{a, t}\right)$ is not locally connected.

Proof. We denote by $B_{a, t}(\infty)$ the immediate basin of $\infty$ for $f_{a, t}$. As the orbit of any point of the circle is dense in $\mathbf{S}^{1}$ (since the rotation number of $\left.f_{a, t}\right|_{\mathbf{S}^{1}}$ is irrational), if the boundary of $B_{a, t}(\infty)$ intersects $\mathbf{S}^{1}$, then it contains the entire circle $\mathbf{S}^{1}$. Hence, according to Lemma 1.5 applied to $C=\mathbf{S}^{1}$ and to $p=\infty$ (recall that $\left.f_{a, t}\right|_{\mathbf{S}^{1}}$ is a diffeomorphism for $a>3$, either $\partial B_{a, t}(\infty) \cap \mathbf{S}^{1}=\emptyset$ or $J\left(f_{a, t}\right)$ is not locally connected. We assume now that $\partial B_{a, t}(\infty) \cap \mathbf{S}^{1}=\emptyset$ and that the Julia set 
is connected. Let $U$ denote the connected component of $\overline{\mathbf{C}} \backslash \overline{B_{a, t}(\infty)}$ containing 0 . Since the closure $\overline{B_{a, t}(\infty)}$ is connected, $U$ is a topological disc. By assumption $\mathbf{D}$ is compactly contained in $U$, so $A=U \backslash \overline{\mathbf{D}}$ is an annulus. If there is no critical point in $A$, the map $f_{a, t}$ is an homeomorphism from $A$ onto itself (since $f_{a, t}$ restricts to a degree one map of $\mathbf{S}^{1}$ ). In this case, $A$ is included in an Herman ring but this contradicts the connectedness of the Julia set. Now, since there is a critical point in $U$, there is a preimage of $\mathbf{D}$ in $U$ because of the Riemann-Hurwitz formula applied to $A$. Let us denote by $D^{\prime}$ this preimage $D^{\prime}=f_{a, t}^{-1}(\mathbf{D}) \backslash \mathbf{D}$. It is homeomorphic to D since for degree reason the non-zero critical point cannot be in $D^{\prime}$. Its closure is contained in $A$ because $\overline{\mathbf{D}}$ is in $A$ and there is no critical point on $\mathbf{S}^{1}$. Therefore $f_{a, t}: A \backslash \bar{D}^{\prime} \rightarrow A$ is a double ramified covering and this contradicts Lemma 1.7.

Lemma 1.7. Let $A$ be an open annulus. There does not exist a disk $D^{\prime}$ such that $\bar{D}^{\prime} \subset A$ with an holomorphic ramified covering $f$ of degree 2 from $A \backslash \bar{D}^{\prime}$ onto $A$.

Proof. Recall that the length-area method allows us to calculate the modulus of an annulus $A$ by the following formula: $\bmod (A)=\inf \left\{\operatorname{area}_{m}(A) \mid m\right.$ is allowable $\}$. Allowable metrics are conformal metrics of the form $m(z)=\rho(z)|d z|$, where $\rho$ is positive and measurable, $m$ is of finite non-zero total area, and every non-zero homotopic curve $\gamma$ in $A$ has length $l_{m}(\gamma)$ belonging to $[1,+\infty]$. It is classical that the infimum is reached only for the euclidean metric i.e., with $\rho(z)=\lambda /|z|$ for some $\lambda>0$ on the round annulus $r \mathbf{D} \backslash \overline{\mathbf{D}}, r>1$.

We assume for contradiction that there exist an annulus $A$, a disk $D^{\prime}$ with $\bar{D}^{\prime} \subset A$ and a ramified double covering $f: A \backslash \bar{D}^{\prime} \rightarrow A$. We will define an allowable metric $m$ on $A$ for which $\bmod (A)=\operatorname{area}_{m}(A)$ but which is zero on $D^{\prime}$ and so cannot be conformally equivalent to the flat one described above; this will give the contradiction. Let $\psi$ be a conformal representation from $A$ to $A_{r}=r \mathbf{D} \backslash \overline{\mathbf{D}}$ and $T \subset A_{r}$ be the straight half-ray $T=\left(\psi(f(c)) \times\left[1,+\infty[) \cap A_{r}\right.\right.$, where $c$ is the ramification point of $f$. Note that $T$ is a half-geodesic for the metric $m_{0}(z)=$ $|d z| /|z|$ on $A_{r}$. Its pullback $\delta$ in $A \backslash \bar{D}^{\prime}$ i.e., $\delta=f^{-1}\left(\psi^{-1}(T)\right)$, is a simple arc. It separates the annulus $A$ into two components: a disk $\Delta$ and an annulus $A^{\prime}$ which is isomorphically mapped by $f$ to $A \backslash f(\delta)$. We define a conformal metric $m$ on $A$ as the pullback of $m_{0}$ on $A^{\prime}$ i.e., $(\psi \circ f)^{*} m_{0}$, extended by 0 on $\Delta$. This measurable metric is also allowable. Indeed, every non-zero homotopic curve $\gamma$ in $A$ has length greater than or equal to $\gamma^{\prime}$, a component of $\gamma \cap A^{\prime}$ that is not zero-homotopic if one adds $\delta$ to it. This implies that $\psi \circ f\left(\gamma^{\prime}\right)$ is not zero-homotopic in $A_{r}$ when one adds $T$ to it. Therefore its length in the flat metric $m_{0}$ is greater than the length of $\mathbf{S}^{1}$. Hence $l_{m_{0}}\left(\psi \circ f\left(\gamma^{\prime}\right)\right)>1$ and since $l_{m}\left(\gamma^{\prime}\right)=l_{m_{0}}\left(\psi \circ f\left(\gamma^{\prime}\right)\right)$, we get that $l_{m}(\gamma) \geq 1$. The metric $m$ satisfies $\bmod (A)=\operatorname{area}_{m}(A)$. Indeed, by definition of $m$ we have that $\operatorname{area}_{m}(A)=\operatorname{area}_{m}\left(A^{\prime}\right)=\operatorname{area}_{\psi^{*} m_{0}}(A)$. On the other side, $m_{0}$ is the flat metric for which the infimum is reached, so $\operatorname{area}_{\psi^{*} m_{0}}(A)=\bmod (A)$. Therefore $\bmod (A)=\operatorname{area}_{m}(A)$, equality that provides the announced contradiction.

\section{THE SECOND EXAMPLE}

This section deals with a different kind of example, namely with rational maps in the family $f_{a}(z)=z^{3} \frac{z-a}{1-a z}$ for $a \in \mathbf{C} \backslash\{ \pm 1,0\}$. It is devoted to the proof of Theorem 1 which is very elementary and can be summarized as follows. We first describe the dynamical properties of the particular map $f_{5}$ and then we perturb it 
inside the family in order to "create" a Cremer point on the boundary of the basin $B_{a}(0)$. We denote by $B_{a}(x)$ the Fatou component containing $x$. When $x$ is an attracting fixed point (like 0 or $\infty$ here), $B_{a}(x)$ is the immediate basin of attraction of $x$. In this case, $B_{a}(x)$ has at most one other preimage that we denote by $B_{a}^{\prime}(x)$.

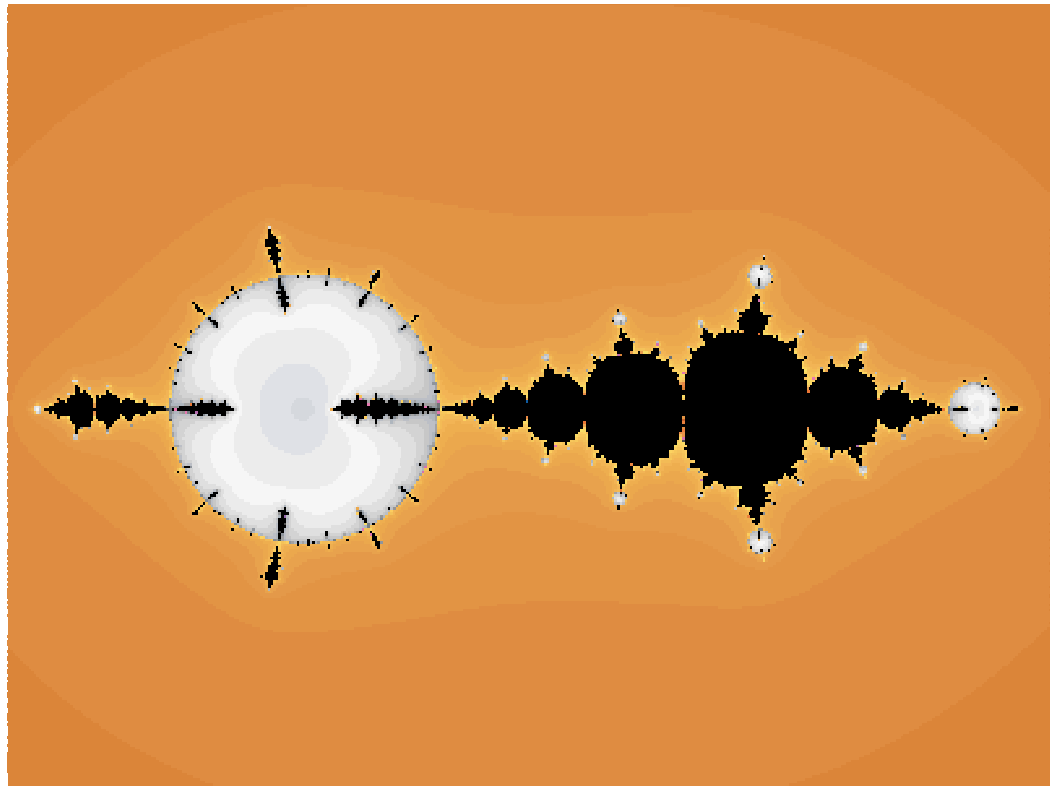

Figure 1. The preimages of $B_{5}(0)$, of $B_{5}(\infty)$ and of the Julia set of the quadratic-like restriction of $f_{5}$ near $p$ and near $1 / p$ are respectively in light color, between dark and light and in black.

Lemma 2.1. The map $f_{5}$ is renormalizable near the parabolic fixed point $p=$ $2-\sqrt{3}$. More precisely, there exist topological disks $U^{\prime}, U$ such that $f_{5}: U^{\prime} \rightarrow U$ is a quadratic-like map and the boundaries $\partial U, \partial U^{\prime}$ are the union of parts of rays and equipotentials in $B_{5}(0)$.

Proof. It is very easy to verify the following properties: The point 1 is a repelling fixed point and the other fixed points are $0, \infty, p=2-\sqrt{3}$ and $1 / p=2+\sqrt{3}$, these last two having multiplier -1 are parabolic; there are only two critical points different from 0 and $\infty$, we denote them by $\omega<1$ and $1 / \omega$. As any parabolic point should attract a critical point, $\omega$ is captured by $p$ and $1 / \omega$ by $1 / p$ (since the circle $\mathbf{S}^{1}$ is invariant). Therefore the Böttcher map $\phi_{5}^{0}$ which conjugates $f_{5}$ near 0 to $z^{3}$, with $\left(\phi_{5}^{0}\right)^{\prime}(0)=1$, is well defined from $B_{5}(0)$ to $\mathbf{D}$. Rays can be defined as usual by $R_{5}^{0}(\theta)=\left(\phi_{5}^{0}\right)^{-1}\left([0,1) e^{2 i \pi \theta}\right)$. As the rays $R_{5}^{0}(0)$ and $R_{5}^{0}(1 / 2)$ are fixed by $f_{5}$, they converge to a repelling or parabolic fixed point (see [DoHu1, Mi1]). If such a fixed ray converges to the parabolic fixed point $p$, it has to go to $p$ through a repelling petal since the union of repelling and attracting petals of $p$ form a neighborhood of the parabolic point (see Mi1). This contradicts the fact that the repelling petals are not fixed but exchanged by $f_{5}$ since the multiplier is -1 . Hence the rays $R_{5}^{0}(0)$ and $R_{5}^{0}(1 / 2)$ converge to the repelling fixed point 1 . Let $V$ denote the bounded connected component of $\mathbf{C} \backslash\left(\overline{R_{5}^{0}}(0) \cup \overline{R_{5}^{0}}(1 / 2)\right)$. We now define the domain on 
which the map $f_{5}$ admits a quadratic-like restriction. We assume, up to replacing $\phi_{5}^{0}(z)$ by $\phi_{5}^{0}(-z)$, that $V$ contains the rays of angle comprised between 0 and $1 / 2$. Hence the rays $R_{5}^{0}(1 / 3)$ and $R_{5}^{0}(1 / 6)$ are included in $V$ and both converge to a preimage of 1 (since they are in the preimage of $R_{5}^{0}(0)$ and $R_{5}^{0}(1 / 2)$ ). There are exactly four preimages of $1: 1,-1$, some $\alpha \in(0,1)$ and $1 / \alpha>1$. Since only $\alpha$ belongs to $V \subset \mathbf{D}$, the two rays converge to $\alpha$.

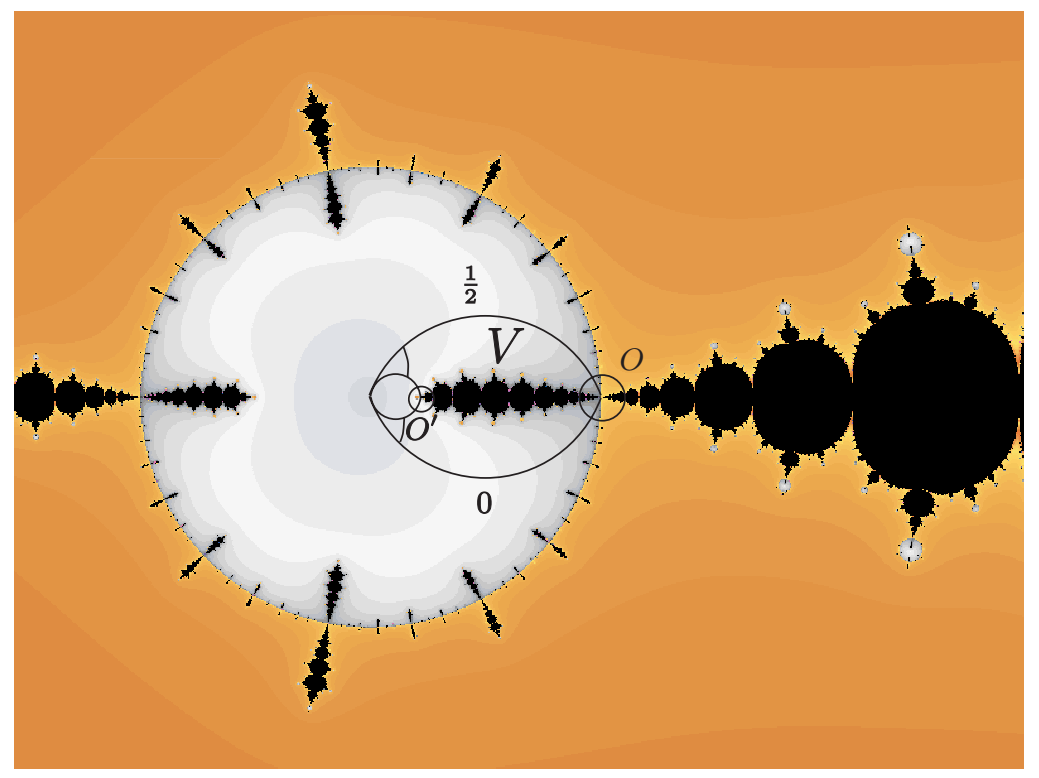

Figure 2. The domain $V$

The open set bounded by (the closure of the four rays) $\overline{R_{5}^{0}}(1 / 3), \bar{R}_{5}^{0}(1 / 6), \overline{R_{5}^{0}}(0)$, $\bar{R}_{5}^{0}(1 / 2)$ contains the critical point $\omega$ since it is a disk whose boundary is mapped with degree two to its image. It follows that the parabolic fixed point $p=2-\sqrt{3}$ also belongs to this disk since the critical point is in a Fatou component containing $p$ in its boundary.

Let $O$ be a small neighborhood of the repelling fixed point 1 such that $f_{5}(O) \supset \bar{O}$. Consider the component $O^{\prime}$ of the preimage of $f_{5}(O)$ containing $\alpha$. For $N$ large enough, the rays $R_{5}^{0}\left(-1 / 3^{N}\right), R_{5}^{0}\left(1 / 2+1 / 3^{N}\right)$ and $R_{5}^{0}\left(1 / 3-1 / 3^{N}\right), R_{5}^{0}\left(1 / 6+1 / 3^{N}\right)$ converge in $O$ and in $O^{\prime}$ respectively. Indeed, $f_{5}$ is geometrically finite (since the critical points are attracted by the parabolic points $p$ and $1 / p$, or are fixed) so its Julia set and, in particular, the boundary of the Fatou components are locally connected (see Wh, TaYi). This implies that the inverse of the Böttcher map $\psi=\left(\phi_{5}^{0}\right)^{-1}: \mathbf{D} \rightarrow B_{5}(0)$ extends continuously to the boundary; in particular the four rays above converge and the landing points are near 1 or near $\alpha$. Now we take $\epsilon>0$ small enough so that the bounded connected component of $\mathbf{C} \backslash \psi\left(\epsilon \mathbf{S}^{1}\right)$ does not intersect $O \cup O^{\prime}$. Let $\Omega$ denote the connected component of $\mathbf{C} \backslash\left(\psi\left(\epsilon \mathbf{S}^{1}\right) \cup O \cup O^{\prime} \cup \gamma\right)$ containing $p$, where

$$
\gamma=\overline{R_{5}^{0}}\left(\frac{1}{3}-\frac{1}{3^{N}}\right) \cup R_{5}^{0}\left(\frac{1}{6}+\frac{1}{3^{N}}\right) \cup \overline{R_{5}^{0}}\left(-\frac{1}{3^{N}}\right) \cup R_{5}^{0}\left(\frac{1}{2}+\frac{1}{3^{N}}\right) .
$$


By construction, the restriction $f_{5}: U^{\prime} \rightarrow U$ is a quadratic-like map where $U^{\prime}=$ $\Omega \cup O \cup O^{\prime}$ and $U=f_{5}\left(U^{\prime}\right)$.

Corollary 2.2. There exists a neighborhood $\Lambda$ of $a=5$, such that for $a \in \Lambda$, the map $f_{a}$ has a fixed point $p(a)$ which is a holomorphic function starting at $p(5)=$ $p=2-\sqrt{3}$. Moreover there exist $U^{\prime}(a), U(a)$ neighborhoods of $p(a)$ which intersect $B_{a}(0)$ such that $f_{a}: U^{\prime}(a) \rightarrow U(a)$ is a quadratic-like restriction.

Proof. Since the derivative of $z \mapsto f_{5}(z)-z$ does not vanish at $z=p$, one can define in a small neighborhood of $a=5$ an holomorphic map $p(a)$, by the Implicit Function Theorem, satisfying $f_{a}(p(a))=p(a)$. Furthermore, quadratic-like maps are stable. More precisely, there exists a smaller neighborhood $\Lambda$ of 5 such that for $a \in \Lambda$ there are disks $U^{\prime}(a)$ and $U(a)$ for which $f_{a}: U^{\prime}(a) \rightarrow U(a)$ is a quadratic-like map. As $\overline{U^{\prime}(a)}$ varies continuously with $a$ (see [DoHu1] $), U^{\prime}(a)$ has to cross $R_{a}^{0}(0)$, since $U^{\prime}$ intersects $R_{5}^{0}(0)$.

Lemma 2.3. There exist parameters $a \in \Lambda$ such that the fixed point $p(a)$ is a Cremer point.

Proof. Let for $\theta \in] \alpha, \beta\left[\subset \mathbf{S}^{1}, h_{\theta}(z)\right.$ be an holomorphic function in $z$, continuous in $\theta$ which for each $\theta$ is not the zero function. It follows from a classical argument (based on the Rouché Theorem) that the set $C$ of $\theta \in] \alpha, \beta[$ such that 0 is a Cremer fixed point for the function $g_{\theta}(z)=e^{2 i \pi \theta} z+z^{2} h_{\theta}(z)$, is a residual set of $] \alpha, \beta[$. Here we can write $\tau^{-1} \circ f_{a} \circ \tau(z)=\lambda(a) z+z^{2} l_{a}(z)$ where $\lambda(a)=f_{a}^{\prime}(p(a)), \tau(z)=z+p(a)$ and $l_{a}(z)$ is holomorphic in $z$, continuous in $a$ and not identically 0 since $f_{a}$ " $(z)$ is not identically zero. Moreover the function $a \mapsto \lambda(a)$ cannot be constant equal to -1 since the set $A$ of parameters $a \in \mathbf{C}$ such that $f_{a}$ has a fixed point of multiplier -1 is a finite set. Indeed, the fixed points of $f_{a}$ in $\mathbf{C}$ are solutions of a polynomial equation $P(a, z)=0$ and the fixed points $z$ with multiplier -1 are solutions of another polynomial equation $Q(a, z)=0$ (which corresponds to $f_{a}^{\prime}(z)=-1$ ). So for $a \in A$ the resultant $R_{a}$ of $P(a,$.$) and Q(a,$.$) vanishes; R_{a}$ is a polynomial in $a$ and so has a finite number of roots, if it is not the zero polynomial. For $a=4$ the fixed points $0, \infty, 1, \frac{3-\sqrt{5}}{2}$ and $\frac{3+\sqrt{5}}{2}$, are all attracting except 1 which is repelling; so $R_{a}$ is not the zero map. As $\lambda$ is a non-constant holomorphic map, $\lambda(\Lambda)$ is an open neighborhood of $\lambda(5)=-1$. Therefore one can define an analytic curve $\gamma \subset \Lambda$ on which $\lambda$ restricts to an homeomorphism onto some small arc $\left\{e^{2 i \pi \theta} \mid \theta \in\right] \alpha, \beta[\}$. We denote by $a:] \alpha, \beta\left[\rightarrow \gamma\right.$ the "inverse" of $\lambda$ satisfying $\lambda \circ a(\theta)=e^{2 i \pi \theta}$. Let $C$ be the set of $\theta \in] \alpha, \beta\left[\right.$ such that $e^{2 i \pi \theta} z+z^{2} l_{a(\theta)}(z)$ has a Cremer point. It is a residual set in $] \alpha, \beta[$ so that there are parameters $a \in \gamma$ satisfying the Lemma.

Corollary 2.4. For any parameter a provided by Lemma 2.3, the Cremer point $p(a)$ is on the boundary of $B_{a}(0)$.

Proof. Let $\phi$ denote the quasi-conformal homeomorphism conjugating $f_{a}: U^{\prime}(a) \rightarrow$ $U(a)$ to a quadratic polynomial $P_{c}: W^{\prime} \rightarrow W$ where $W^{\prime}, W$ are the neighborhoods of $J\left(P_{c}\right)$ images of $U^{\prime}(a)$ and $U(a)$ respectively by $\phi$ (see DoHu2]). By Corollary [2.2. the immediate basin $B_{a}(0)$ crosses $U^{\prime}(a)$; let $x$ be a point in $B_{a}(0) \cap U^{\prime}(a)$. Its image $y=\phi(x)$ lies in $W^{\prime}$. Its backward orbit under $P_{c}$ accumulates every point of $J\left(P_{c}\right)$, so that there exists a sequence $\left(y_{n}\right)$ of iterated preimages by $P_{c}$ converging to the Cremer fixed point $\phi(p(a)) \in J\left(P_{c}\right)$. Hence the sequence $x_{n}=\phi^{-1}\left(y_{n}\right)$ converges to $p(a)$ and stays in $B_{a}(0)$. Indeed, for $a \in \Lambda$ consider the curve 
$\gamma_{a}=\bar{R}_{a}^{\infty}(1 / 3) \cup \bar{R}_{a}^{\infty}(1 / 6)$ image by $\iota(z)=1 / z$ of the curve $\delta_{a}=\bar{R}_{a}^{0}(1 / 3) \cup \bar{R}_{a}^{0}(1 / 6)$. For $a_{0}=5, \delta_{a_{0}}$ is a Jordan curve that surrounds the pole $1 / a_{0} \in[0,1]$ (since it is not in $\left.U\left(a_{0}\right)\right)$. Hence $\gamma_{a_{0}}$ surrounds the zero $a_{0}$ and, since $O$ is small, $\gamma_{a_{0}}$ does not intersect $U\left(a_{0}\right)$. This situation is stable for $a$ near $a_{0}$ (since the point 1 is still repelling). Therefore, $\gamma_{a}$ separates $B_{a}^{\prime}(0)$ from $U(a)$. For this reason $x_{n} \in B_{a}(0)$ and $p(a)$ is on the boundary of $B_{a}(0)$.

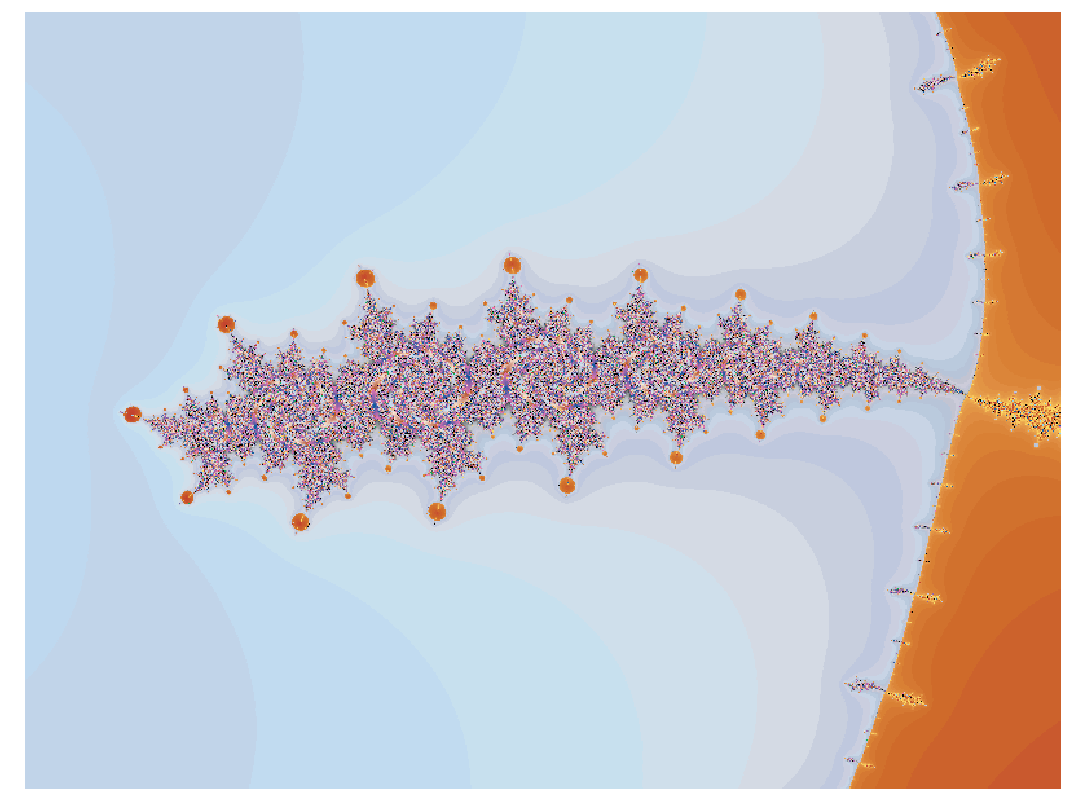

FiguRE 3. Julia set around the fixed point $p(a)$ for $a$ near 5

Lemma 2.5. For any parameter a given by Lemma 2.3, the Julia set $J\left(f_{a}\right)$ is connected but not locally connected.

Proof. The Julia set is connected since it satisfies clearly the condition of Shishikura's Theorem: A rational map, which has at most one weakly repelling fixed point (i.e. repelling or with multiplier exactly 1), has a connected Julia set. Moreover it follows directly from Lemma 1.5 applied to the compact $C=p(a)$ that $J\left(f_{a}\right)$ is not locally connected.

Lemma 2.6. For any parameter a given by Lemma 2.3, the map $f_{a}$ is a genuine rational map.

Proof. The maximum principle easily implies the following topological property for the Julia set $J$ of a polynomial. Let $K$ be any connected set contained in $J$, let $U$ be a neighborhood of $J$ and $z_{1}, z_{2} \in J \backslash K$; then there exists a path $\gamma \subset U \backslash K$ joining $z_{1}$ to $z_{2}$. As we show next, this property is not satisfied here by $f_{a}$, so $f_{a}$ is a genuine rational map. We consider the curve $l_{a}=\bar{R}_{a}^{0}(0) \cup R_{a}^{0}(1 / 2)$. For $a=5$ it separates $B_{a}^{\prime}(\infty)$ from $B_{a}(\infty)$ (see Lemma 2.1). This curve is stable in the neighborhood $\Lambda$ of 5 since the point 1 remains repelling. Therefore the curve $l_{a}$ still separates $B_{a}^{\prime}(\infty)$ from $B_{a}(\infty)$. Let $x \in \partial B_{a}^{\prime}(0) \cap \partial B_{a}(\infty)$, for instance on 
$\partial B_{a}(\infty)$ (considering the rays in $B_{a}(\infty)$ ). Let $x^{\prime}$ be its preimage on $\partial B_{a}^{\prime}(\infty) ; x$ and $x^{\prime}$ cannot be connected by a path in the complement of $K=\partial B_{a}(0)$ (which is a connected subset of the Julia set) since the curve $l_{a}$ separates them (any path should cross $l_{a}$ and therefore $\left.\partial B_{a}(0)\right)$.

\section{OTHER EXAMPles?}

Note that the two examples we have described "come from polynomials" (in different ways). So it would be very interesting to find an example without any references to polynomials.

Question 3.1. Does there exist a rational map $f$, that has no connection with polynomials, such that $J(f)$ is connected but not locally connected?

Another related question would be to prove that non-local connectivity does not come from Fatou components :

Question 3.2. Does there exist a rational map $f$ such that $J(f)$ is connected, not locally connected and the boundary of every Fatou component is locally connected.

Recall that if $J(f)$ is locally connected, the boundary of every Fatou component is locally connected (see $\mathrm{Wh}$ ).

\section{ACKNOWLEDGMENTS}

I would like to thank the referee for very pertinent remarks and very useful suggestions. I am very grateful to A. Douady for several very interesting discussions on the Ghys, Herman, Douady and Sullivan example and in particular for Lemma 1.7 and Proposition 1.6. I would also like to thank I. Liousse for instructive discussions on Herman's work.

\section{REFERENCES}

[AnMa] J. Anderson, B. Maskit, On the local connectivity of limit set of Kleinian groups, Complex Variables Theory Appl. 31 (1996), 177-183. MR.1423249(98a:30055)

[DoHu1] A. Douady, J. H. Hubbard, Etude dynamique des polynômes complexes, Publications mathématiques d'Orsay 1984.

[DoHu2] A. Douady, J. H. Hubbard, On the dynamics of polynomial-like mappings, Ann. scient. Éc. Norm. Sup. 18 (1985), 287-343. MR0816367 (87f:58083)

[Do] A. Doundy, Disques de Siegel et anneaux de Herman, Séminaire Bourbaki, 39 (19861987) $\mathrm{n}^{\circ}$ 677, 151-172. MR0936853 (89g:30049)

[Gh] E. GHYs, Transformations holomorphes au voisinage d'une courbe de Jordan, C.R.Acad. Sc. Paris, t.298 (1984), 385-388. MR0748928 (86a:58081)

[He] M. Herman, Conjugaison quasi-symétrique des difféomorphismes du cercle à des rotations et applications aux disques singuliers de Siegel, I., manuscript, http://www. math.kyoto-u.ac.jp/mitsu/Herman/qsconj2

[Mi1] J. Milnor, Dynamics in One Complex Variable, Vieweg 1999, 2nd edition 2000. MR.1721240 (2002i:37057)

[Mi2] J. Milnor, Local Connectivity of Julia Sets: Expository Lectures, pp. 67-116 of "The Mandelbrot set, Theme and Variations" ed.: Tan Lei, LMS Lecture Note Series 274 , Cambr. U. Press 2000. MR.1765085 (2001b:37073)

[Min] Y. Minsky, On rigidity, limit sets, and end invariants of hyperbolic 3-manifolds, J. Amer. Math. Soc. 7 (1994) no. 3, 539-588. MR1257060 (94m:57029)

[McM1] C. McMullen, Complex Dynamics and Renormalization, Annals of Mathematics Studies, Princeton University Press, Princeton 1994. MR1312365 (96b:58097)

[McM2] C. MCMullen, Local connectivity, Kleinian groups, and geodesics on the blowup of the torus, Invent. Math. 146 (2001), 35-91. MR1859018 (2004e:30068) 
[Ro] P. Roesch, On local connectivity for the Julia set of rational maps, Ann. Math. (To appear).

[So] D. SoREnsen, Describing quadratic Cremer point polynomials by parabolic perturbations, Ergodic Theory Dynam. Systems 18 (1998), no. 3, 739-758. MR1631760 (99e:58146)

[Su] D. Sullivan, Quasiconformal homeomorphisms and dynamics I, solution of the FatouJulia problem on wandering domains, Ann. Math. 122 (1985), 401-418. MR0819553 (87i:58103)

[Sh] M. SHISHIKURA, The connectivity of the Julia set of rational maps and Fixed points, Preprint IHES, Bures-sur-Yvette, 1992.

[TaYi] L. TAN, Y. YIn, Local connectivity of the Julia set for geometrically finite rational maps, Science in China (Serie A) 39 (1996), 39-47. MR1397233 (97g:58142)

[Wh] G. Whyburn, Analytic topology, AMS Colloquium Publications 28, 1942. MR0007095 $(4: 86 \mathrm{~b})$

[Za] S. ZAKERI, In Shahyad, a volume dedicated to Siavash Shahshahani on the occasion of his 60th birthday, 2002.

UMR Paul Painleve, University of Lille 1, Cité scientifique - BÂtiment M2, 69655 Villeneuve d'Ascq Cedex, France

E-mail address: roesch@math.univ-lille1.fr 